\title{
Design on Multi Parameter Monitoring System for Fire Disaster Environment Based on Wireless Sensor Network
}

\author{
Bo Chang ${ }^{1,}$, , Xinrong Zhang ${ }^{2, \mathrm{~b}}$, XV Bao-guo ${ }^{3, \mathrm{c}}$ \\ ${ }^{1}$ Faculty of Computer Engineering, Huaiyin Institute of Technology, Huai'an, Jiangsu, China \\ ${ }^{2}$ Faculty of Electrical Engineering, Huaiyin Institute of Technology, Huai'an, Jiangsu, China \\ ${ }^{3}$ School of Internet of Things Engineering, JiangNan University, wuxi 214122,China \\ aemail:mmm33534@sohu.com, bemail:nn33@163.com, ${ }^{c}$ email: xbg@jiangnan.edu.cn
}

Keywords: fire disaster environment, wireless sensor network (WSN), multi parameter, monitoring

\begin{abstract}
Aiming at the problems existing in the fire disaster monitoring, such as lower automation level, the detection point difficult to change and expand, and lower cost performance compared with similar monitoring products, etc., an intelligent monitoring system for fire environmental parameters is proposed based on wireless sensor networks. The system uses ZigBee network to reduce data traffic. The wireless network with microprocessor CC2530 as the core control device can collect real-time data such as temperature, humidity and smoke concentration in the fire environment, these processed data will be sent to the monitoring center for storage and display, to achieve a unified data management and network routing monitoring function. The monitoring results have shown that this system is stable, high reliable in data transmission and easy to use, and is suitable for environmental monitoring occasions which are inconvenient to connect directly.
\end{abstract}

\section{Introduction}

Fire monitoring system is the most effective and safe guarantee for early warning of fire, prevention of fire, and timely realization of firefighting [1]. There are many kinds of fire detectors. According to the detector's structure and shape, it is divided into two categories: linear and point type [2]. According to the difference of fire parameters, it can be divided into temperature sensing, smoke sensing, photosensitive, gas and composite types, etc. [3]. The combination of wireless sensor network (WSN) technology and intelligent fire detection technology can solve many disadvantages of traditional wired mode, and realize the fire detection's wireless, network, intelligence, which has become a new research direction in the field of fire safety [4].

At present, the automatic fire monitoring system mainly adopts wired connection mode, which brings the problems that the system has many consumables, high cost, large power consumption, low anti-interference ability, complex design, construction and maintenance. Using hard wire connection, the line is easy to aging or corrosion, resulting in the system itself fault rate is high, high false positive rate. Wireless network fire monitoring system can solve the above problems well, and has a great development prospect in fire prediction. WSN technology is widely used in military, environmental monitoring [5], engineering safety [6, 7], the agricultural greenhouse [8], livestock and poultry farms and food processing [9] and other fields. At present, the wireless security monitoring system used in foreign countries mainly uses ZigBee Technology [10, 11], uses intelligent routing algorithm, and uses multi-hop Mesh structure consisting of about 50 nodes.

In view of the specific characteristics of the fire site, applying WSN technology based on microprocessor fast, flexible control and the characteristics of PC monitoring and management functions, a fire scene monitoring system was designed, mainly composed of low power wireless sensor network node through the ZigBee network [12]. Through the arrangement of detection nodes in the laboratory area, monitoring the status of each parameter variable in the operation, the actual monitoring effect of the system is tested. The experimental results show that the system can effectively monitor the temperature, humidity and smoke concentration in the fire scene. 


\section{Requirement Analysis and Structure Design of Monitoring System}

Firstly, carrying out the demand analysis of fire monitoring system application market, according to the user's needs, different types of sensors, such as smoke sensing, temperature sensing and photoelectric sensors etc., are installed and completing fire detection by the detector. Then, the detector controller keeps detecting the state of the probe to judge the data transmitting over the sensor to find the fire in time or the malfunction information. The collected data is transmitted and aggregated in real time using the low-cost wireless communication. Next, on the basis of information detection, data processing and fusion are provided, which provide the basis for control decision to form control output.

The technical requirements of the system are as follows. The system adopts the control and communication equipment based on the ZigBeeCC2530, which is suitable for mass production and for the needs of all kinds of engineering. The probe and wireless interface circuit use $4.5 \mathrm{~V}$ power supply, supported by three battery-powered. The working voltage of controller is AC220V $\pm 10 \%$, $50 \mathrm{~Hz} \pm 1 \mathrm{~Hz}$, and its power consumption in surveillance state is equal to or less than 20W. A controller can connect with about 50 detectors, employing two groups of channels for communication, and it fully meets the demands of various types of building monitoring. The transmission distance of open space should greater than $100 \mathrm{~m}$, and the internal building situation should greater than $30 \mathrm{~m}$. The temperature of the environment should be in the scope of $0 \sim+45^{\circ} \mathrm{C}$, and the relative humidity should be less than or equal to $95 \%$.

The system mainly includes three function components: the monitoring central computer, gateway node and sensor nodes. The whole system adopts one-to-more monitoring structure. The system structure is shown in Fig.1.

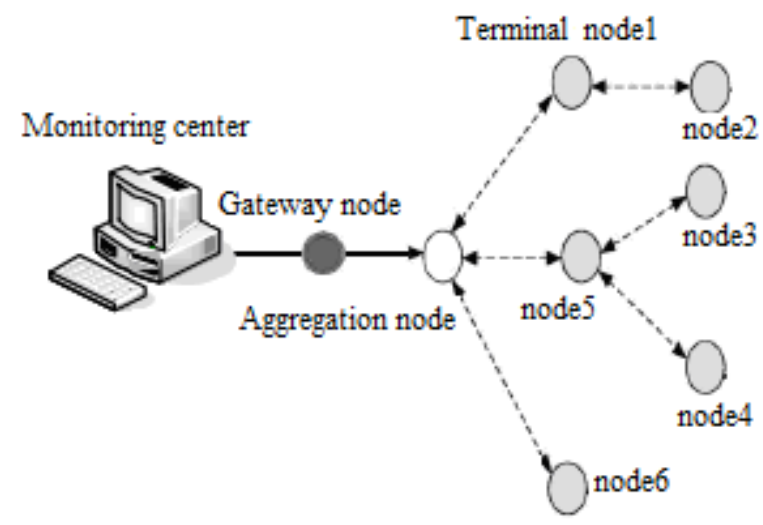

Fig.1. Structure diagram of system

The fire detector nodes are deployed inside the monitoring area, which can not only sense and collect the data of temperature, humidity and smoke concentration on the environment, but also can communicate with the data. As the gathering node, the gateway node connects with the computer through the RS-232 serial port, and these nodes can form a network with multi hop routing function. Running a special background monitoring software from the PC of the monitoring center, receiving the uploaded data from gateway node and processing them, then displaying the data and combining them, all of which are from data center and then into the database. If the fire message is found, the database judge it, once a fire occurs, fire alarm can be sent immediately.

\section{Hardware Design of Hardware Platform for Fire Environment Monitoring System}

Hardware Design of Monitoring Node. The hardware block diagram of the fire detection node is shown in Fig.2, which consists of a microcontroller chip, a series of fire detection sensors and power supply modules. At the scene of the fire environment monitoring application background, the design of sensor nodes should focus on the factors of low cost, low power consumption, stability and reliability. 


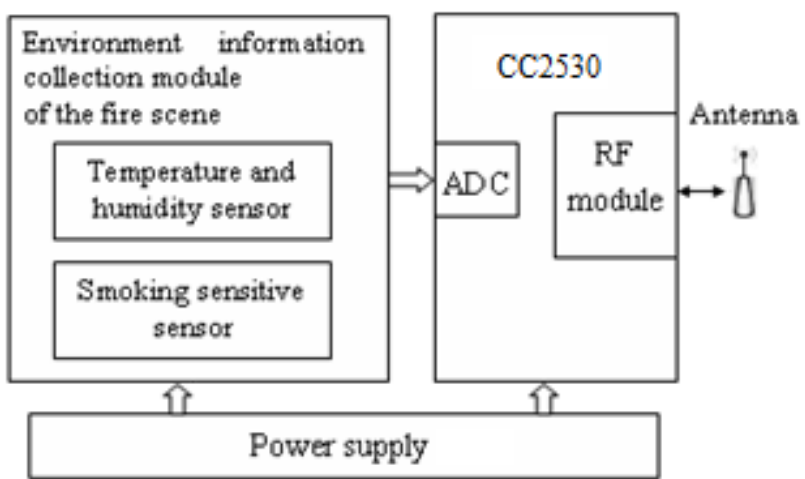

Fig.2. Hardware block of fire detection node

For CC2530, due to the rich resources, powerful functions, simple wiring and less peripheral circuit, the system can improve the reliability while reducing power consumption. Smoke sensors, temperature and humidity sensors are suitable for early fire, and can produce alarms in time, but it is not suitable for outdoor detection and not allowed to open fire occasions. The detector and the wireless interface circuit use $4.5 \mathrm{~V}$ power supply, so three dry batteries power the supply module.

The Selection of the Fire Detector Sensor. The principle of fire detection is to detect the various physical phenomenon and the chemical reaction produced by the burning material. According to the principle of fire detection, dozens of fire detectors have been developed. Identifying different environmental parameters can determine different fire types or early fire background parameters. After introducing the network structure, as long as the communication reliability is guaranteed, the multi parameter system can be considered as a mature fire detection system. The detector integrated with temperature, humidity and photoelectric smoke sensor, combined with the software design, can automaticly detect temperature, humidity and smoke concentration of the fire scene. The smoke sensor adopts chip MS5100, a semiconductor gases smoke sensor, and it has the advantages of small size, high sensor sensitivity, good stability, fast response time, and having strong sensitivity to smoke, hydrocarbons and oxides, etc. The node uses ordinary 2 AA alkaline batteries, without additional power stabilizer. Temperature and humidity sensor module uses chip SHT11, which has the advantages of digital output, avoid debugging, avoid calibration, high precision, low energy consumption, auto sleep and being completely submerged in the water. The measurement resolution of the chip temperature and humidity can respectively reach 14 bit and 12 bit, and the power consumption is very low, the resting state is only $0.3 \mu \mathrm{A}$.

The Design of Gateway node. Considering the specific requirements and cost accounting, the system can still use CC2530 to design gateway nodes, similar to the composition and design of sensor nodes. The function of gateway node is to conduct data calibration, fusion, and send data to the monitoring central PC. Considering the system requirements and cost, the design of gateway node still use the CC2530, whose composition and design is similar to the terminal sensor. On the one hand, Gateway node receives the data from network sensor and process them, and then send them to the monitoring central PC. On the other hand, Gateway node receives the commands sent by the central monitoring computer, processes the instructions accordingly, and releases monitoring tasks to the terminal nodes. Both the sending and receiving data adopt double-ended (differential) signals in the RS-485 interface, which can effectively overcome the common mode interference and improve the data transmission rate and transmission distance. Therefore, in order to meet the requirements of the fire environment monitoring system, the RS-485 with long communication distance and reliable data transmission is selected as the backbone type of the network.

\section{Monitoring System Software Platform Design}

The Software Process of Sensor Node. The work process of a sensor node includes power-on self test of system, data acquisition, data receiving and sending and power management, etc. In the node software design, these functions, including the device initialization, configuring the network, 
starting the network, and realizing the self-organization network of multiple sensor nodes distributed in the fire environment, are completed by calling the API function provided by the ZigBee protocol stack. The workflow of a node is shown in Fig.3.

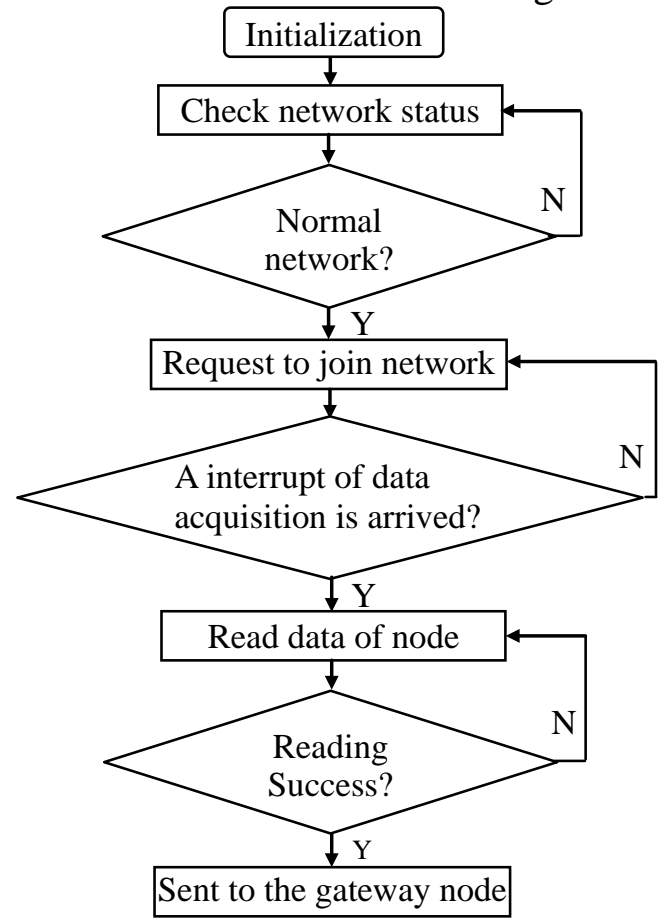

Fig.3. Software flow of the sensor node

Node begin to work after detector power is turned on, that is, the system initialize the hardware circuit and parameters, and when power-on startup is completed, the program enters the main loop state. In the main loop, the detector will complete the automatic networking, timing detection, detection, and data transceiver.

The Design of Main Software Interface. Adopting modular design approach, the monitoring system software consists of the four parts: the management module of system user, system parameter setting module, data monitoring module, setting module of history data, which are shown in Fig.4. Through the monitoring center PC, the staff can observe parameter changes of temperature, humidity and smoke concentration of the fire environment. The specific design method is that in $\mathrm{C}++$ Builder IDE, window application based on the visualization components is to be built, named SCOMMN, and in the building process, all steps keep the default, adding controls in the main form.

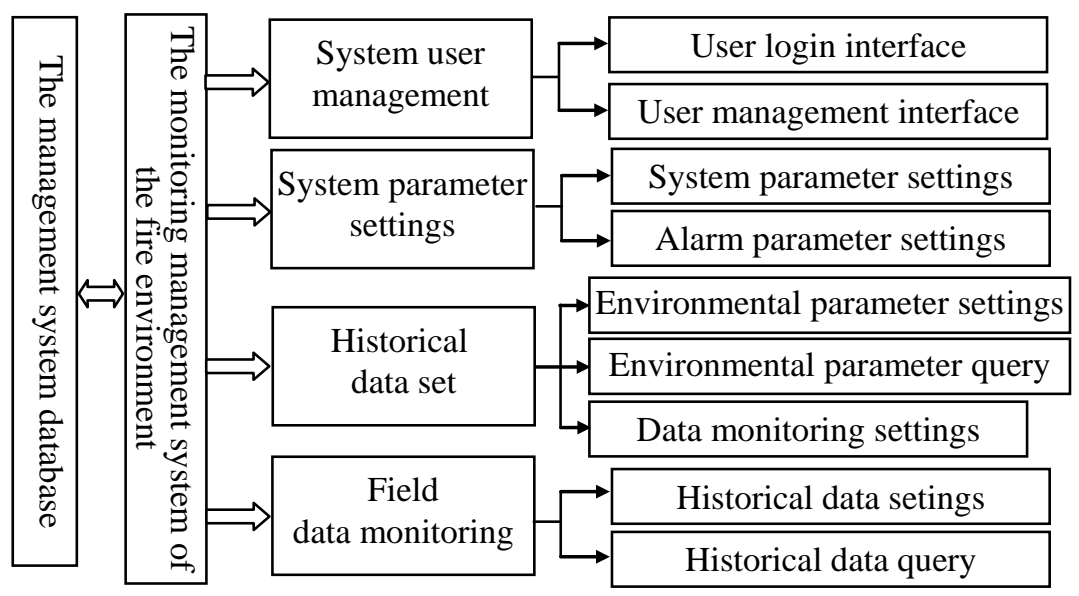

Fig.4. Diagram of main operation interface

\section{Monitoring results and data analysis of fire environment}

According to system design requirements, three nodes were assembled. ZigBee has excellent low 
power consumption and reliable transmission capability. Therefore, as long as the network topology is properly designed, the system can fully meet the needs of fire detection. The two fire detectors (denoted as \#1, \#2) were installed in the annular wire lift $3 \mathrm{~m}$ away from $2 \mathrm{~m}$, the other detector as a gateway node was placed in $15 \mathrm{~m}$ away, connected to the PC running background monitoring software via the RS-232 interface. Installing temperature, humidity and smoke sensors in the two fire detectors, through a comparison between the data collected by the detector nodes and the data uploaded to the monitoring center, the data transfer accuracy of the wireless detector nodes and the sensitivity of fire detectors can be verified. Table 1 is part of the data collected on the \#1 detection node.

Table 1. Monitoring data tables of fire environment parameters

\begin{tabular}{|c|c|c|c|c|c|c|c|c|c|}
\hline \multirow{2}{*}{$\begin{array}{l}\text { Measurements } \\
\text { (\#1 node) }\end{array}$} & \multicolumn{9}{|c|}{$\begin{array}{l}\text { Monitoring and measured values of fire after a certain period } \\
\text { of time (s) }\end{array}$} \\
\hline & 10 & 20 & 40 & 80 & 100 & 140 & 160 & 180 & 200 \\
\hline $\begin{array}{l}\text { Temperature } \\
\text { monitoring value }\left({ }^{\circ} \mathrm{C}\right)\end{array}$ & 24.5 & 26.8 & 28.7 & 30.6 & 33.7 & 37.9 & 45.4 & 56.8 & 68.9 \\
\hline $\begin{array}{l}\text { Temperature } \\
\text { measured value }\left({ }^{\circ} \mathrm{C}\right)\end{array}$ & 24.6 & 26.9 & 28.9 & 30.8 & 33.9 & 38.1 & 45.7 & 56.9 & 69.2 \\
\hline $\begin{array}{l}\text { Humidity } \\
\text { monitoring value (\%) }\end{array}$ & 32 & 32 & 33 & 34 & 34 & 35 & 35 & 36 & 36 \\
\hline $\begin{array}{l}\text { Humidity } \\
\text { measured value (\%) }\end{array}$ & 33 & 33 & 34 & 35 & 35 & 36 & 36 & 37 & 37 \\
\hline $\begin{array}{l}\text { Smoke concentration } \\
\text { monitoring value }(\%)\end{array}$ & 15 & 19 & 25 & 37 & 49 & 68 & 84 & 94 & 96 \\
\hline $\begin{array}{l}\text { Smoke concentration } \\
\text { measured value }(\%)\end{array}$ & 16 & 21 & 27 & 39 & 50 & 70 & 87 & 95 & 97 \\
\hline
\end{tabular}

From the above experimental data, it can be seen that the wireless fire detector node can accurately detect the temperature, humidity and smoke concentration parameters. It is verified by experiments that the monitoring system of fire environmental parameters can be automatically constructed by wireless communication, can monitor the fire parameters in real time, and can transmit the monitoring data to the monitoring center PC through the gateway node and deal with the data.

\section{Conclusions}

This paper analyzes the current development of fire detection technology, puts forward a fire monitoring system based on wireless sensor network. Applied the advanced wireless sensor network technology to the fire detection, a kind of compound parameter fire detector with wireless communication function is designed and developed, which can detect simultaneously three parameters of temperature, humidity and smoke concentration when the fire happens, so as to improve the accuracy of the fire alarm. Compared with the single fire parameter detector, this system reduces the possibility of false alarm and lost alarm. The experimental results show that the system can correctly respond to three parameters of temperature, humidity and smoke concentration, can judge easily the occurrence of fire, and has broad application prospects.

\section{References}

[1] J Zhang, W Li, Z Yin, S Liu, X Guo. Forest fire detection system based on wireless sensor network [J]. Chinese Journal of Sensors \& Actuators, 2013, 694-697 (3) :369-374.

[2] Young Neil.Fire, Dctection, Alarm Systems [J].Fire Prevention and Fire Engineers Journals. 2004, 4:53-55.

[3] A.Keller, M.Ruegg, M.Forster, et al. Open Photo Acoustic Sensoras Smoke Detector.Sensors and Actuators.2005, 104:1-7. 
[4] Soonil Nam, Leo P Donovan, Jieon Grace Kim. Establishing. Heat Detectors' Thermal Sensitivity Index through Bench-Scale Tests[J]. Fire Safety Journal.2004, (39):191-197.

[5] M.E.Layman.Wireless Mobile Fire InsPections[J]. Fire Engineering.2005, 158(11):110-111.

[6] Jaime Lloret, Miguel Garcia, Diana Bri and Sandra Sendra. A Wireless Sensor Network Deployment for Rural and Forest Fire Detection and Verification [J].Sensors 2009, 9 (11): 8722-8746.

[7] ZhaiYuwen.Themonitoring system ofwireless data-transmission for water supply station [ J]. Transaction of Jilin Chemical Institute, 2000, 11(2): 39-42.

[8] Zeng X Z, Liu G, Zheng D P, et al. Study and development of a field information acquisition system based on wireless technique[C]//Actual Tasks on Agricultural Engineering, Opatija, CROATIA, 2006: 371 377.

[9] Sun Zhongfu, Du K M, Han H F, et al. Design of a telemonitoring system for data acquisition of livestock environment [C] //Livestock Environment VIII-Proceedings of the 8th International Symposium, Iguassu Falls, Brazil: ASABE, 2008: 995 1000.

[10] Beijing. Using WSN for Forest Fire Monitoring and Rescue Application [J]. Microcomputer Information, 2008 , 24 (10):169-171.

[11] M Hefeeda, M Bagheri. Forest Fire Modeling and Early Detection using Wireless Sensor Networks [J]. Ad Hoc \& Sensor Wireless Networks, 2008, 7 (3):169-224.

[12] Pottie G J, Kaiser W J. Wireless integrated network sensors[J].Communications of the ACM, 2000, 43(5): 51-58. 